\title{
Zum Labyrinth moralischer Gefühle zu Reichtum
}

\author{
Martin Schürz
}

Angenommen: 30. Juni 2021 / Online publiziert: 13. September 2021

(C) Der/die Autor(en) 2021

Zusammenfassung Daten zu Vermögen, Einkommen und Ausgaben einerseits und Narrative zu Reichtum andererseits stehen in einem losen Verhältnis zueinander. Menschen positionieren sich in der Vermögensverteilung an falscher Stelle und unterschätzen die ungleiche Vermögensverteilung deutlich. In Narrativen zu Reichtum ist zudem von Leistung, Habgier, Verdienst und Großzügigkeit die Rede.

Zur Klärung der Bedeutung von Narrativen zu Reichtum ist daher eine Bezugnahme auf Gefühle von Menschen wichtig. Adam Smith hat in seiner Theorie der ethischen Gefühle wesentliche moralpsychologische Überlegungen zu Sympathie angestellt. Ich konzentriere mich auf seine Beobachtung, dass arme Menschen Reiche bewundern und argumentiere, dass ein solches Gefühl der Bewunderung rational fragwürdige Narrative stärken kann. Bestimmte Narrative zu Reichtum korrespondieren zwar nicht mit empirischen Mikrodaten aus Haushaltserhebungen, aber sie entsprechen reichtumsfreundlichen Gefühlen.

Schlüsselwörter Vermögen · Adam Smith · Ungleichheit · Legitimation · Reiche Menschen 


\section{The labyrinth of moral emotions on affluence}

Abstract Data on wealth, income and consumption and narratives on wealth concentration are only in a vague manner connected. People do not know their position in wealth distribution and underestimate wealth inequality by far.

Narratives on the rich refer to effort, greed, merit and generosity. Exploring the relevance of narratives on the rich needs a focus on emotions. Adam Smith considered in his "Theory of moral sentiments" moral psychological ideas. In this study I will concentrate on his observation, that poor people admire rich people. Such an emotion can strengthen questionable narratives. Some narratives on the rich do not correspond with empirical microdata from household surveys but relate to sympathetic emotions towards the rich.

Keywords Wealth $\cdot$ Adam Smith $\cdot$ Inequality $\cdot$ Legitimation $\cdot$ Affluence

\section{Einführung}

Die Rechtfertigung von Reichtum ist ein komplexes Unterfangen. Bei der Legitimation von Unterschieden im Arbeitseinkommen wird oft der Markt als Letztinstanz betrachtet, weil dort entschieden werde, was ein gerechtfertigtes Einkommen darstellt. Und manche ÖkonomInnen betrachten dies als hinreichend (siehe Hayek 2005). Doch bei Reichtum fällt der Markt vorab als Legitimationsinstanz aus. Denn viel an Vermögen erreicht den Markt überhaupt nicht. Immobilien etwa bleiben im Familienverbund über Generationen.

$\mathrm{Zu}$ dem traditionellen reichenbegünstigenden politischen Instrumentarium zählt heute insbesondere privater Eigentumsschutz. In der Wirtschaftspolitik sind Unternehmersubventionen, Unternehmenssteuersenkungen, Abschaffung von Vermögensund Erbschaftssteuern Maßnahmen, die seit den 1980er-Jahren an Bedeutung gewonnen haben.

Doch auch reichenfreundliche Narrative spielen eine wichtige Rolle bei der Verteidigung der verfestigten privaten Vermögenskonzentration. Für deren Wirksamkeit ist die Beachtung moralischer Gefühle entscheidend. Moralische Gefühle hegen Menschen zu normativen Fragen, d.h. zu dem, was sein soll. Hierzu zählen etwa Fragen, wer soll was verdienen, wer soll weniger habgierig sein, wer soll mehr Gutes tun und wer möge den eigenen Neid bekämpfen, anstatt Gerechtigkeitsforderungen aufzustellen, aber auch wer soll bewundert werden. In ökonomische Analysen, fließen immer nichtökonomische Werturteile ein" (Gabriel 2020, S. 299).

Thomas Piketty schrieb zu Anfang seines Werkes Kapital und Ideologie, dass das Bedürfnis menschlicher Gesellschaften, ,ihren Ungleichheiten einen Sinn zu geben, mitunter auch jenseits aller Vernunft“", unüberwindbar sei (Piketty 2020, S. 50). Die Formulierung jenseits aller Vernunft weist daraufhin, dass Narrative, Werte und Gefühle in sozialwissenschaftlichen Untersuchungen hinkünftig stärker beachtet werden müssen.

Piketty richtet seinen Blick auf Ideologien der Ungleichheit. Sein Fokus auf Rechtfertigungen der Ungleichheit ist eine entscheidende Erweiterung gegenüber 
seinem ersten Werk (siehe Piketty 2014). In einer Demokratie werden Rechtfertigungen für hohe Einkommens- und Vermögensungleichheit benötigt. Die Frage, was ist eine exzessive, respektive eine gesellschaftlich problematische Ungleichheit, findet keine Antwort in Statistiken. Es gibt keinen optimalen Wert des GiniKoeffizienten, aber auch keinen richtigen Anteil des Top-1\% am gesamten Vermögen. Antworten auf diese Fragen müssten demokratisch ausgehandelt werden. An ökonomische ExpertInnen können sie nicht delegiert werden.

Soziale Ungleichheit ist für Piketty daher „keine wirtschaftliche oder technologische, sie ist eine ideologische und politische Ungleichheit" (Piketty 2020, S. 21). Dies ist eine weitreichende Behauptung. Ungleichheit könnte ökonomisch determiniert und zudem auch ideologisch begründet sein. Bei Piketty ist aber Ideologie das zentrale Charakteristikum von Ungleichheit. Piketty argumentiert, dass Gesellschaften nicht ohne Ideologien auskommen, mit denen sie ihren Ungleichheiten einen Sinn zu geben versuchen. Es komme entscheidend auf den historisch jeweils hegemonialen Diskurs zu Ungleichheit an.

Piketty will hierbei ,jeder Ideologie der Vergangenheit eine Chance geben “ (Piketty 2020, S. 24). Zu diesen Ideologien zählt er trifunktionale, sklavenhalterische, kolonialistische, proprietaristische, kommunistische und neoproprietaristische gesellschaftliche Diskurse. Diese grobflächige Einteilung ist in ihrer historischen Grenzziehung nachvollziehbar. Sie macht die Bedeutung unterschiedlicher Ideologien in verschiedenen Gesellschaftsformationen mit unterschiedlichen Institutionen und Ordnungen klar. Ideologie bildet aber nicht unbedingt einen historisch gut abgrenzbaren Untersuchungsgegenstand (Schürz 2021). So gibt es Ideologien, die sich zwar geschichtlich in ihrer Bedeutung unterscheiden, deren Vorhandensein sich aber über Jahrhunderte erstreckt. Das Narrativ zur Habgier der Reichen ist vermutlich das beste Beispiel. Es reicht von der Antike bis in die Gegenwart.

Gerechtigkeitsprinzipien spielen eine geringere Rolle als ein diffuses Ungerechtigkeitsempfinden in der Bevölkerung. Wenn nur ganz wenige Menschen ein Milliardenvermögen haben - weltweit sind es etwa nur wenige tausend Menschen und diese als Leistungsträger verstanden werden, so mutieren sie gleichsam zu Übermenschen. Aber dies wertet implizit alle anderen als Minderleister ab. Und es macht das Gerechtigkeitsprinzip der Leistung zu einer Schimäre.

Es ist eine gefühlte Ungerechtigkeit, welche für Menschen zentral ist. Und selten sind es rationale Gerechtigkeitsprinzipien, welche maßgeblich sind in den Narrativen zur Akzeptanz sozialer Ungleichheit (siehe Shklar 2014). In ihrem Handeln folgen nur die wenigsten Menschen detaillierten philosophischen Gerechtigkeitskonzepten. Die meisten werden von Gefühlen geleitet, die sie kaum hinreichend reflektieren. Zum Zusammenhang von Gefühlen und Politik gibt es viele Analysen (etwa Frevert 2013; Nussbaum 2014, 2016; Shklar 2013).

Die Moralpsychologie analysiert die moralischen Empfindungen von Menschen (siehe Shklar 1992, 2014). Göran Therborn schrieb jüngst in seinem Essay zu „Dynamics of inequality“ in der „New Left Review“: „,The rich evoke a number of different emotions and reactions: not only a sense of injustice, but also of resentment, fascination, envy and admiration, as idols of luxury, glamour and success." (Therborn 2017, S. 16). Diese Gefühlskomplexität spielt eine bislang unterschätzte Rolle bei konkreten Gerechtigkeitsurteilen und ideologischen Rechtfertigungen von 
Reichtum (Schürz 2019). Erst das Beachten oder das Evozieren von Gefühlen in der Bevölkerung macht wirtschaftspolitische Programme mehrheitsfähig.

Auch beim Thema der Ungleichheit geht es spezifisch darum ,die feingliedrigeren Legitimierungsprozesse in der empirischen Forschung sichtbar zu machen und in die Theoriediskussion zurückzuführen." (Yildiz et al. 2018, S. 157). Die Legitimitätsbildung sei stärker im Alltag zu verorten.

Narrative müssen nicht wahr sein, um ihre Wirkung zu entfalten. Narrative können intuitiv überzeugen oder ungehört verpuffen. Ebenso gibt es Narrative, die eher zu trösten suchen. TV-Seifenopern über die Reichen bilden nicht die Realität ab, sondern bieten sich als Projektionsfläche für Sehnsüchte ihrer SeherInnen an. Auch dienen Narrative der Ungleichheit oft der Ablenkung von anderen Fragestellungen (Smith Ochoa 2019).

Moralische Wertungen zu Reichtum finden sich schon beim Begriff von Vermögen. Vermögen vermag. Menschen sprechen von den Reichen als den besseren Leuten oder bezeichnen sie als Super-reiche. Ihre Rede von den Höherstehenden deutet auf eine moralische Hierarchie zu Reichtum. Georg Simmel schrieb im Jahr 1900 in seiner Philosophie des Geldes, Reichtum gelte:

sogar als eine Art moralischen Verdienstes ....; was sich nicht nur in dem Begriff der Respectability oder in der populären Bezeichnung wohlhabender Leute als ,,anständiger“, als ,, besseres Publikum“ ausdrückt, sondern auch in der Korrelaterscheinung: daß der Arme behandelt wird, als hätte er sich etwas zuschulden kommen lassen, daß man den Bettler im Zorne davonjagt, daß auch gutmütige Personen sich zu einer selbstverständlichen Überlegenheit über den Armen legitimiert glauben. (Simmel 1989, S. 275)

Eine „,selbstverständliche Überlegenheit“ von Vermögenden wird in keinen Narrativen von Übermenschen aufbereitet, aber sie mag sich als Überzeugung in der Gefühlswelt von Menschen durchaus finden. Moralische Gefühle müssen daher bei einer Analyse der Narrative zu Reichtum eine besondere Beachtung erfahren.

Adam Smith (1723-1790), der Gründervater der Ökonomie, hatte in seinem Hauptwerk, An Inquiry into the Nature and Causes of the Wealth of Nations, 1776 festgestellt:

Das Ansehen der Reichen ist zwar in jedem Zeitalter der Gesellschaft groß, aber am größten ist es wohl in den rohesten Zeiten derselben, sofern sie nämlich eine bedeutende Vermögensungleichheit aufzuweisen hat. (Smith, 2009, S. 726).

Und er bezog Reichtum auf Armut, d.h. er betrachtete Vermögenskonzentration nicht isoliert:

Überall, wo viel Eigentum ist, da ist auch große Ungleichheit. Für einen sehr reichen Mann muß es wenigstens fünfhundert Arme geben und der Überfluß bei den wenigen setzt die Dürftigkeit bei der Menge voraus. Der Überfluß der Reichen erweckt den Unwillen der Armen, die oft durch Mangel und durch Neid dazu getrieben werden, die Besitzungen der ersteren anzufallen .... Es erfordert 
also das Erwerben eines wertvollen und großen Eigentums die Einsetzung einer bürgerlichen Regierung. (Smith, 2009, S. 725).

Zumeist wird Adam Smith als Begründer der freien Marktwirtschaft porträtiert. Sein Werk wird populärwissenschaftlich rezipiert über die Idee der unsichtbaren Hand. Dies ist eine verkürzende Sichtweise (siehe Sen 2010; Evensky 2005; Obermeier 2019). Smiths moralpsychologische Überlegungen aus seiner Theorie der ethischen Gefühle, welche er 17 Jahre vor seinem Hauptwerk „Wealth of Nations“ geschrieben hat, gehen weit über den ökonomischen Fachbereich hinaus. Sie bleiben wegweisend für empirische Untersuchungen von Reichtum.

Von einem zentralen Narrativ in der Theorie der ethischen Gefühle zu sprechen, wäre aber verfehlt. Es gibt verschiede Narrative in diesem wichtigen Buch und ich stelle nicht den Anspruch, den zentralen identifizieren zu wollen (für andere Lesarten siehe Evensky 2005; Obermeier 2019). Was mich intellektuell antreibt, ist die Suche nach Reichtum stabilisierenden Gefühlen. Mein Erkenntnisinteresse gilt nicht der Charakterisierung reicher Menschen, sondern jenen Gefühlen, welche Menschen den Reichen zu deren materiellen Vorteil entgegenbringen.

Sympathie ist ein griechisches Wort. Es bezeichnet die Fähigkeit des Nachempfindens von Gefühlen von anderen Menschen, Sympathie bei Smith ist , unser Mitgefühl mit jeder Art von Affekten “ (Smith 2010, S. 8). Im Begriffsverständnis von Adam Smith ist es insbesondere die Disposition, angesichts der Freude von Mitmenschen selbst auch Freude zu empfinden. Denn Menschen sind nicht nur egoistisch. Ihnen eignet auch Sympathie. Menschen empfinden Reichtum: ,als etwas Großes und Schönes und Edles auf dessen Erlangung wohl all die Mühen und Ängsten wert ist, die wir so gerne auf sie zu verwenden pflegen. "(Smith 2010, S. 295).

Adam Smith wusste, dass Menschen Reichtum nur selten philosophisch analysieren. Nicht rationale Überlegungen stehen im Zentrum der Beobachtungen von Reichen und Mächtigen. Ausgestattet mit Einbildungskraft, seien Menschen von Reichtum fasziniert. Dies ist reichtumsstabilisierend, obwohl es auch in den Augen von Adam Smith einen Irrtum darstellt. Doch so können die Reichen die Illusionen nutzen, welche Menschen über sie hegen, um ihre Privilegien zu behalten. Reiche können ihren übermäßigen Reichtum an ein Gefühl der Bewunderung des Restes der Bevölkerung knüpfen. Dafür benötigen sie keine rationalen Begründungen, sondern den Weg weist ihnen unsere Einbildungskraft. Offensichtlich ist die Vermögenskonzentration ungerecht, doch wenn Menschen das Leben des Top-1\% als paradiesisch imaginieren und daran eine Freude haben, wird die Vermögenskonzentration verfestigt.

Reichtum als Leitbild einer Gesellschaft weist auch auf diesen Zusammenhang. Viele Menschen lieben das Narrativ von kühnen Unternehmern ohne Bezugnahme auf unterbezahle Arbeiter oder die eigenen unbelohnten Anstrengungen. Steve Jobs, der Studienabbrecher, der die Computerwelt revolutionierte, war ein Lieblingsbeispiel eines Selfmade Milliardärs. Aber auch der Werdegang von Jeff Bezos und Elon Musk wird gerne zitiert, um zu zeigen, dass es Einzelne schaffen können. Ein Charakteristikum dieser Menschen sei ihre Fähigkeit, spektakuläre Innovationen zu generieren. Ihr Erfindungsgeist wird so bunt gemalt, dass die materielle und rechtliche Unterstützung des Staates nicht bloss in den Schatten gestellt, sondern 
völlig vergessen wird. Übersehen werden dabei die mit Hungerlöhnen abgespeisten ArbeiterInnen und übersehen wird der Staat mit seinen vielfältigen Unternehmenssubventionen und der steuerfinanzierten Bereitstellung von Infrastruktur.

Nun gibt es in der Historie auch kritische Narrative zur Habgier der Reichen. Und in der Philosophiegeschichte findet sich eine lange Tradition der moralischen Verurteilung der Reichen (Aristoteles 1999; Brown 2017).

Adam Smith hatte einen Lehrstuhl für Moralphilosophie inne und in der Theorie der ethischen Gefühle finden sich zahlreiche moralisierende Urteile. So mahnt er etwa: „Habgier überschätzt den Unterschied zwischen Armut und Reichtum.“ (Smith 2010, S. 235) und er verurteilt den Reichtum ,als ungeheuere und mühsam konstruierte Maschine ersonnen, um ein paar wertlose Bequemlichkeiten für körperliches Wohlbefinden zustandezubringen. " (Smith 2010, S. 294). Dies ist eine klassische moralisierende Aburteilung von Reichtum und stellt eine in der Geschichte der Philosophie vertraute Linie dar. Bewunderung der Reichen über Sympathie hingegen weist einen interessanten und komplexen Pfad.

Die Insinuation der moralischen Verkommenheit sehr vermögender Menschen wäre allein durch das empirische Faktum von großzügigen reichen Philanthropen widerlegt. Reiche seien protzig, meinte zwar Aristoteles. Sie haben ein anmaßendes Lebensgefühl, weil es mit ihren Erfahrungen korrespondiert: denn stünden nicht die Weisen vor den Toren der Reichen und nicht umgekehrt?

Doch selbst wenn Habgier ein typischer Wesenszug von reichen Menschen wäre, so handeln auch manche arme Menschen gierig. Eine spezifische Zuordnung der Gier zu Vermögenden ist nicht begründet. Kein Armer will zudem wohl reich werden, um gierig sein zu können. Es ist demnach kein funktionales Gefühl für die soziale Akzeptanz von Vermögenskonzentration. Bei Wohltätigkeit wäre dies anders. Arme Menschen können von Reichtum träumen, um ihn danach zu verschenken.

Bei Menschen treffen demnach viele und widerstreitende Gefühle zu Reichtum aufeinander. Interessant für meine Untersuchung sind wohlwollende Gefühle, welche ärmere Menschen den Reichen entgegenbringen. Erst reichenfreundliche Gefühle tragen zur Stabilisierung der Vermögenskonzentration bei. Für diese Gefühle sind Narrative zum Verdienst der Reichen nützlich. In diesem Text führe ich Überlegungen aus meinem Buch Überreichtum (2019) weiter.

Smith Ochoa (2019) beschreibt über eine Diskursanalyse wie steigende Ungleichheit über eine ordoliberale storyline verharmlost wird. Seine Schlussfolgerung ist, dass die subjektiven Wahrnehmungen selten berücksichtigt werden. Auf Basis von Mikrodaten folge ich dieser Argumentationslinie und will zeigen, dass die reichtumsabsichernden Narrative nicht zu den vorhandenen Vermögensdaten passen, dass sie aber sehr wohl zu reichtumsfreundlichen Gefühlen in einem Naheverhältnis stehen können.

\section{Verdienst der Reichen}

In Debatten zu Reichtum spielt die Frage des Verdienstes stets eine zentrale Rolle. Adam Smith diagnostizierte, dass die Reichen in jedem Zeitalter ein hohes gesellschaftliches Ansehen genießen. Und auch beim Moralisieren über das Skandalon 
eines Überreichtums geht es um die Frage des Verdienstes (siehe Schürz 2019). Viele Narrative suchen hierbei die Tugendhaftigkeit oder den herausragenden Charakter sehr reicher Menschen zu betonen.

Reichtum kann auf vielfältige Weise zu begründen gesucht werden. Verdienst ist ein vager Terminus und daher vielseitig verwendbar. Vom Verdienst der Reichen zu sprechen, öffnet semantische Ambivalenzen. So kann Reichtum über Leistung als verdient begründet werden, aber auch über Wohltätigkeit. Im Fall der Philanthropie eines reichen Erben spielt dessen eigene Leistung keine Rolle in der Begründung, Reichtumsentstehung und Reichtumsverwendung werden getrennt. Eine solche Trennung in Narrativen ist aber arbiträr, weil die Formen der Vermögensverwendung nicht unabhängig von der Art ihrer Entstehung gesehen werden können: der Drogenbaron, der Schulen in seinem Umfeld finanziert, kreiert trotz der sinnvollen Vermögensverwendung ein gesellschaftliches Problem.

Verdienst ist jedenfalls eine moralisch und politisch umkämpfte historische Begriffskategorie. Sie steht bei der Operationalisierung im Alltag oft in einem Naheverhältnis zu Arbeit, ihre Messung erfolgt meist an der Höhe des Erwerbseinkommens. Dies engt die Betrachtung vorab heimlich auf Fragen der Erwerbsarbeit ein. Die Vermögenskonzentration wird aber getrieben durch Erbschaften und Schenkungen (Fessler und Schürz 2018).

Was über die Zeit gleich zu bleiben scheint, ist der Wunsch reicher Menschen nach einer verdienten superioren sozialen Stellung. Doch was sich wandelt ist das Verständnis von Verdienst. So hielten die aristokratischen Reichen im 19. Jahrhundert ihre Sonderstellung für verdient, aber sie verstanden darunter den Verdienst der Herkunft. Und dies bedeutete eben, dass sie nicht arbeiten mussten. Die USamerikanischen, nicht-aristokratischen Reichen im 19. Jahrhundert wandten sich etwa gegen das allgemeine Wahlrecht. Sie waren Eigentümer riesiger Immobilien und hätten gerne dazu noch die Ehre des englischen Adels gehabt. Der englische Adel hielt aber Reichtum, dessen Quellen Arbeit und Handel waren, für weniger maßgeblich als den eigenen Ruhm auf dem Schlachtfeld und den aristokratischen Lebensstil. Um Leistung ging es den müßiggängerischen Reichen in den USA wohl auch nicht und trotzdem sahen sie die eigenen Privilegien als verdient an. Die Ehre der englischen Aristokraten war ihnen verwehrt. Doch die Verachtung von Arbeit dürfte auch eine Kernüberzeugung dieser Reichen gewesen sein. Den aristokratischen Lebensstil suchte der wohlhabende Teil des Bürgertums auf der Basis ihres Reichtums zu imitieren.

Im 20. Jahrhundert wurde nicht mehr die Erblinie betont, sondern - insbesondere in den USA - ein Narrativ von sozialen Aufstiegsmöglichkeiten. Ein Alleingang beim sozialen Aufstieg brachte mehr an Reputation als das Halten eines privilegierten sozialen Status über Generationen. Als Königsweg zu verdientem Reichtum wurde hartes Arbeiten propagiert. Die Tugenden der Disziplin, des Fleißes und des Durchhaltevermögens waren legitimatorisch wichtig für den Reichtum im 20. Jahrhundert. Aber auch Sparsamkeit, Innovation und Risikoorientierung galten als Meriten, die den Weg zu Reichtum weisen. Von den für Reichtum entscheidenden Vermögenstransfers, wie Erbschaften und Schenkungen, die auch ohne Fleiß oder andere Tugenden zufallen, konnte so abgesehen werden. Die Ansicht, dass Vermögen auf Ersparnissen aus dem Arbeitseinkommen ruhen, widerspricht ökonomischen Daten 
(siehe Fessler und Schürz 2017). Doch nichtsdestotrotz folgt sie einer moralischen Hierarchie, welche Verzicht und Genügsamkeit als Tugenden betont.

Die interessantesten Romane waren, nach dem Urteil von Adam Smith, jene, wo sich die unglücklichen Schicksale von tugendhaften Mächtigen wenden. Der in Nöte gestürzte Held erhebt sich mittels Weisheit und Tapferkeit aus der unglücklichen Lage und kehrt zurück in die herausragende Stellung. Es bringe Menschen zum Schwelgen, wenn die Reichen an ihren naturgemäßen Platz zurückkehren. Bei Smith war es noch ein Heimkehren, welches den Leserinnen gefiel und nicht der Wechsel sozialer Klassen.

Beide Narrative, einerseits zu verdienten Geburtsprivilegien und andererseits zur sozialen Mobilität passen heute nicht mehr. In einer Demokratie soll es keine feudalistischen Privilegien geben, und die Aufstiegschancen sind limitiert und verschlechtern sich in vielen Ländern. Smith hatte die ständische Ordnung noch für natürlich gehalten und sich gefreut, dass die Rangeinteilung in der „Gesellschaft sicherer auf dem klaren und handgreiflichen Unterschied der Geburt und des Vermögens als auf dem unsichtbaren und oft unsicheren Unterschied des Weisheit und Tugend rühren würden." (Smith 2010, S. 368).

\section{Tugend der Reichen}

Bernard Mandeville (1670-1733) hatte die zentrale These seiner Bienenfabel (1714) kurz und bündig private Laster, öffentliche Vorteile genannt. Mandevilles These von den privaten Lastern, die sich in öffentliche Tugenden verwandeln, ist in der Ökonomik als ,Mandeville-Paradoxon“"bekannt geworden. Mandeville sah die Gesellschaft gespalten in zwei soziale Gruppen, fleißige Arme und genusssüchtige und korrupte Reiche. Aber er rehabilitierte die Luxusorientierung der Reichen und zeigte die engen Grenzen der Tugend auf, weil diese einerseits oft keine ist und andererseits die Vorteile des Luxus übersehen werden. Mandeville polemisierte sogar gegen herzlose Reiche, die ihr Vermögen wohltätig spendeten, anstatt es den rechtmäßigen Erben verdienterweise zukommen zu lassen. Adam Smith überzeugten die Gedanken von Mandeville wenig (Smith 2010, S. 597).

Bei Smith ist Sympathie die Basis der Billigung. Sympathie darf, wie zuvor beschrieben, nicht mit Wohlwollen verwechselt werden. Daher würde Mandevilles Polemik gegen die Tugend bei Smith ins Leere zielen. Und auch das zynisch anmutende Narrativ von den lasterhaften Vermögenden, welche zurecht die tugendhaften Armen verdrängen, hat sich im historischen Diskurs nur als Polemik oder Satire etabliert. Als Legitimation für Privilegien ist es hingegen nicht nützlich.

Adam Smith erwartete bei den Armen ,einen beträchtlichen Grad von Tugend.“ (Smith 2010, S. 97). Zu diesem Urteil gelangte er aber nicht, weil er meinte, dass die Armen die besseren Menschen seien, sondern weil diese nicht die Macht haben, sich über die Gesetze zu stellen. Arme seien allein deswegen ehrlicher, weil sie berufliche Fähigkeiten benötigen und echte Dienstleistungen erbringen müssen. Schmeichelei und Falschheit, zentrale Eigenschaften an fürstlichen Höfen, bringen ihnen nichts. Tugenden entstehen demnach aus Schwäche. ,Moralisten ermahnen uns zur Mildtätigkeit und zum Mitleid. Sie warnen uns vor dem Blendwerk der Grö- 
ße. Dieses Blendwerk ist freilich so mächtig, daß die Reichen und Großen allzuoft den Weisen und Tugendhaften vorgezogen werden." (Smith 2010, S. 367).

Im 4. Deutschen Armuts- und Reichtumsbericht wurde unterschieden zwischen: „,nur 'Reiche(n) und im Wortsinne Vermögende(n), die ihre besonderen Möglichkeiten zur Ausübung sozialer Verantwortung nutzen" (Bundesministerium für Arbeit und Soziales 2013, S. 412). Während die Reichen bloß an ihren persönlichen Vorteil denken, würden die Vermögenden Wertvolles für die Gemeinschaft tun. Der Reiche handle demnach amoralisch und der Vermögende trotz Profitorientierung gemeinwohlorientiert. Bei der Tugendhaftigkeit würde es sich entscheiden, wer zu den Vermögenden und wer nur zu den Reichen zählt. Den Armen wäre diesem Gedankengang folgend die Möglichkeit, tugendhaft zu sein, übrigens vorab verbaut. Sie können Steuern seltener vermeiden oder hinterziehen. Die Lohnsteuer wird vom Gehalt abgezogen und die Mehrwertsteuer beim Einkauf kann auch nicht vermeiden werden. Es geht in vielen Narrativen folglich nur um eine moralische Unterscheidung innerhalb der kleinen Gruppe der Vermögenden, zwischen tugendhaften und lasterhaften Reichen.

Theodor W. Adorno hatte im Aphorismus „Tugendspiegel“ seiner Minima Moralia eine Verschränkung von Reichtum und Tugend erkannt und diese kritisch gesehen:

Reichtum als Gutsein ist ein Element des Kitts der Welt; der zähe Schein solcher Identität verhindert die Konfrontation der Moralideen mit der Ordnung, in der die Reichen recht haben, während zugleich andere konkrete Bestimmungen des Moralischen als die vom Reichtum abgezogenen nicht konzipiert werden konnten. (Adorno 2003, S. 211).

Nun sind reiche Menschen moralisch nicht wertvoller als arme Menschen. An diese einfache Einsicht muss immer erneut erinnert werden in einer Welt von gütigen Philanthropkapitalisten (siehe Callahan 2017). Oft sind sie unglücklich und handeln moralisch fragwürdig. Experimente des Psychologen Piff belegen etwa so ein ethisch problematisches Verhalten (Piff 2014). Doch wichtiger scheint mir die Einsicht von Adam Smith, dass wir über unsere Einbildung ihres Glücks auch die Frage nach ihrer Tugend oftmals affirmativ beantworten.

\section{Bewunderung der Reichen}

Adam Smith diagnostizierte, dass die Bewunderung von Menschen füreinander recht einseitig ausfalle. Die meisten Menschen himmeln eher Vermögende als Arme an: „Häufig sehen wir die achtungsvolle Aufmerksamkeit der Welt stärker auf die Reichen und Vornehmen sich richten, als auf die Weisen und Tugendhaften. Häufig sehen wir, daß die Laster und Torheiten des Mächtigen weit weniger verabscheut werden als die Armut und Schwäche des Unschuldigen." (Smith 2010, S. 94). Armut wird verabscheut, die Armen werden verachtet und Reichen wird nahezu eine Apotheose zuteil. Menschen neigen dazu, ,, die Reichen und Mächtigen zu bewundern und beinahe göttlich zu verehren und Personen in ärmlichen und niedrigen Verhältnissen zu verachten" (Smith 2010, S. 93). 
Der Gedankengang von Adam Smith in der Theorie der ethischen Gefühle geht folglich von einer zeitdiagnostischen Beobachtung aus. Doch verbunden mit seiner Konzeptualisierung von Sympathie ist er weitreichender. Arme imaginieren in ihrer Fantasie den Zustand der Reichen als einen idealen. Deshalb bewundern sie die Reichen. Sie bilden sich ein, dass das Glück der Reichen nahezu paradiesisch ist.

Bei der Lektüre von Hochglanzgazetten, auf Instagramm bei rich kids, in TV Serien zu reichen Familien nehmen Menschen auch heute sympathisierend Anteil an den Höhen und Tiefen im Leben der Reichen, während sie etwa das Elend von Flüchtlingen negieren bzw. sogar als Privileg beurteilen können.

Wir können bezweifeln, dass diese Feststellung von Smith für die Gefühle aller Menschen zutrifft. Sogar in einem empirischen Fragebogensetting wären Bewunderung und Verachtung nur indirekt und unzureichend abzufragen. Eigene Gefühle werden oft verkannt, missverstanden und manchmal auch nur ungern einbekannt. Wer Reiche insgeheim bewundert, mag dieses Bejubeln vielleicht aus moralischen Gründen an sich selbst verurteilen oder öffentlich bestreiten, dass dem so sei. Doch unbestreitbar ist, dass es Menschen gibt, die Reiche bewundern und dass diese Bewunderung viel weniger Aufmerksamkeit in öffentlichen Verteilungsdebatten bekommt als etwa Gier und Neid. Die in der sozialen Hierarchie nach oben gerichtete Bewunderung ist eben funktional. Sie erlaubt den Menschen, sich mit ungerechten Herrschaftsverhältnissen abzufinden.

Um sich mit den Härten der Realität zu arrangieren, sind Tagträume in unser aller Leben zuweilen vonnöten. Tagträume zum Glanz der Vermögenden, zu ihrem kühnen Aufstieg, ihren unternehmerischen Wagnissen und ihrem luxuriösen Lebensstil erlauben, den eigenen bescheidenen Alltag leichter zu ertragen. Solche Tagträume werden von Narrativen zum Verdienst der Reichen und zu deren Wohltätigkeit befeuert. Die Figur des innovativen Unternehmers, großzügigen Spenders, genialen Erfinders, solitären Gewinners im Wettbewerb oder fürsorglichen Fortführers der Familientradition ist nur in Verbindung mit Sympathie für die Legitimation von Reichtum hilfreich. Ohne diese könnte eine Vermögens- oder Erbschaftssteuer einfach entlang von Gerechtigkeitsprinzipien legitimiert werden.

Doch es ist das Gefühl der Bewunderung, welches eine Einfallsschleuse für verschiedene legitimatorische Narrative zur verdienten gesellschaftlichen Privilegierung liefert. Menschen werden möglicherweise von herrschaftsabsichernden Ideologien und von Bullshiting (Harry Frankfurt) irregeführt, aber sie suchen auch selbst nach Narrativen, die zu ihrem Wohlwollen den Reichen gegenüber passen.

$\mathrm{Ob}$ das Wohlwollen, welches reichen Menschen entgegengebracht wird, ihrerseits auf reichenfreundlichen Narrativen basiert, oder ob die Sympathie erst die gesellschaftliche Wirkung von reichenfreundlichen Narrativen ermöglicht, ist ungeklärt. Kausalität kann keine gefunden werden, aber das Korrespondenzverhältnis ist evident.

Smith führt ein weiteres wichtiges Argument an, welches seine These von der Bewunderung der Reichen stärkt. Er meint, dass wir weit stärker dazu geneigt sind, die Freude als das Leid anderer Menschen mitzufühlen. Es ist angenehmer, mit glücklichen Menschen mitzufühlen als mit unglücklichen mitzuleiden. Nur Neid und Missgunst können gegen dieses freundliche Gefühl wirken. 
Ein allfälliger Neid auf das Leben der Reichen verschönert das eigene Leben aber nicht. Neid lässt verbittern. Bewunderung kann hingegen bereichern. Warum sollten Menschen daher ohne Notwendigkeit ein galliges Gefühl wählen? Und eine Erfordernis für Neid gibt es wohl auch nicht. Die soziale Distanz zwischen Milliardären und den übrigen Menschen ist enorm. Für das Aufkommen von Neid haben die meisten Menschen kaum Gelegenheit, da Reich und Arm in getrennten Sphären leben.

Bereits Bernard Mandeville hatte die Notwendigkeit von sozialer Nähe für das Aufkeimen von Neid präzise erkannt:

Wenn einer, der zu Fuß gehen muß einen großen Mann beneidet, weil er sich einen Wagen mit sechs Pferden hält, so wird dies nie mit der Heftigkeit geschehen oder ihm den Verdruß bereiten, wie einem Manne, der selbst einen Wagen hat, aber sich bloß vier Pferde leisten kann. (Mandeville 2014, S. 178).

Reichtum erlaubt zwar ostentativ zur Schau gestellten Luxus (Wiesing 2015). Und Luxus bietet eine Möglichkeit zu protzen und aus gutem Grund anzugeben. Der gute Grund sei, dass dies anderen Menschen erleichtere, mit uns zu sympathisieren. Denn nur um Wohlergehen geht es bei Vermögenden nicht. Auch die Reichen glauben nicht, dass , in einem Palast ihr Magen besser oder ihr Schlaf gesünder sei als in einer Hütte. " (Smith 2010, S. 78). Aber ein Palast eignet sich eben weit besser zum eitlen Prahlen als ein kleines Haus.

Smith begrüßte demnach die Funktionalität einer Täuschung. Nur ob dieses Irrtums der Menschen werde der Boden bearbeitet, werden Häuser gebaut und werden die Wissenschaften vorangetrieben. Bei Adam Smith gehen eine moralische Ablehnung des Strebens nach Reichtum und eine Betonung der ökonomischen Nützlichkeit dieses Reichtumstrebens zusammen.

In der Haushaltserhebung zu Einkommen, Vermögen und Ausgaben, die im Europäischen System der Zentralbanken regelmäßig durchgeführt wird (Household Finance and Consumption Survey, Household Finance and Consumption Survey (HFCS) (europa.eu)) finden sich auch diverse Variablen zu Einstellungen zu Reichtum und Werturteilen zu Ungleichheit. Im Folgenden werde ich einige Datenergebnisse aus unserer Erhebung HFCS-Austria in Beziehung zu den moralpsychologischen Gedanken von Adam Smith setzen.

Das Median-Haushaltsvermögen beträgt in Österreich 83.000€. Zur Veranschaulichung der Vermögensunterschiede sollen Unterschiede bei der Körpergröße dienen. Der Median bei der Körpergröße liegt bei etwa 1,73 m. Folgen wir dieser Analogie der Körpergröße, so gelangen wir für den reichsten Menschen in Österreich mit einem Vermögen von $37 \mathrm{Mrd}$. $€$ auf einen Wert von $773 \mathrm{~km}$. Es ist demnach keine Vermögenswelt von Kleinen und Großen, sondern ein fast unvorstellbare Distanz zwischen Arm und Reich.

Menschen müssten den Reichtum der Vermögenden erst einmal angemessen erkennen, um Narrative rational beurteilen zu können. Doch dies tun sie nicht, wie Abb. 1 zeigt. Sie haben keine Ahnung, dass die untere Hälfte nur einen minimalen Anteil von unter $3 \%$ am gesamten Vermögen hat. Und sie schätzen auch den Reichtum der Vermögenden nicht annähernd richtig ein. 


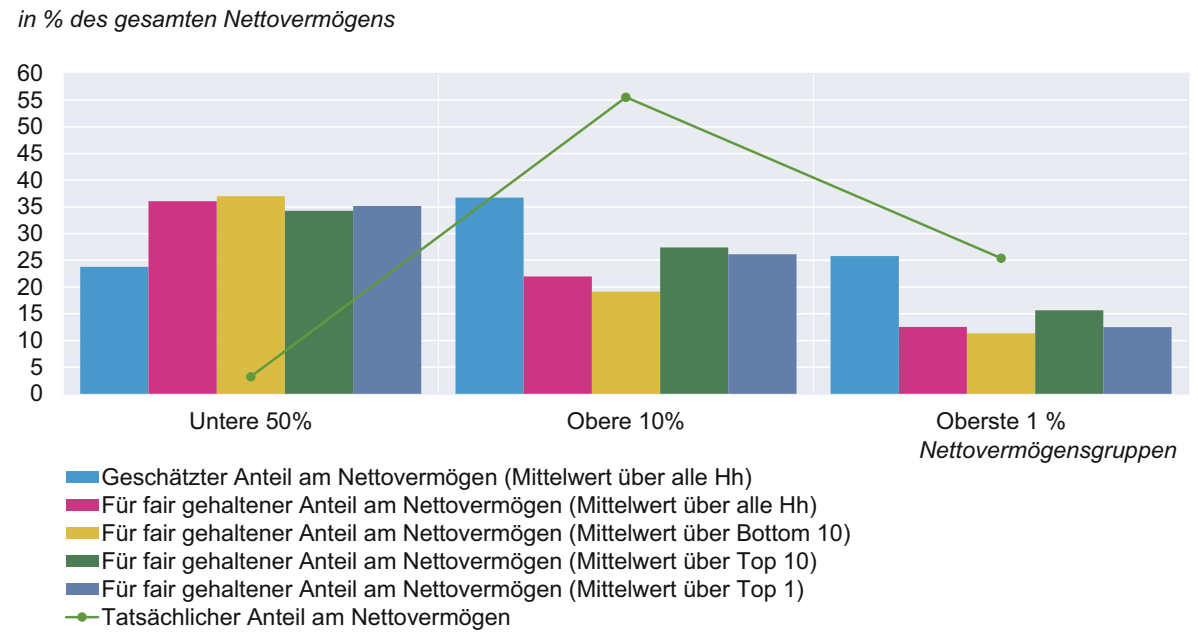

Abb. 1 Verteilung von Nettovermögen. Quelle: HFCS Austria 2014

Trotz der enormen Unterschiede beim Vermögen wollen Menschen nun aber keine gleiche Gesellschaft. Doch sie hätten gerne eine weit egalitärere Gesellschaft. Sie würden dafür optieren, dass die Unteren $50 \%$ einen weit größeren Anteil und die Top-1\% einen viel kleineren Anteil (12,5\%) am gesamten Vermögen halten, als diese tatsächlich haben. Der gemessene Anteil der Vermögendsten $1 \%$ in Österreich liegt zwischen 23 und $40 \%$, je nachdem, wie statistisch mit fehlenden Angaben und einer geringeren Teilnahmebereitschaft der Reichen bei der Erhebung und mit der Unsicherheit der Informationen aus Reichenlisten umgegangen wird. Interessanterweise würden auch die Vermögenden selbst einen weit geringeren Anteil am gesamten Vermögen für fair halten. Gerechtigkeitsurteile und Positionierung in der Vermögensverteilung fallen teilweise auseinander, was auch für eine stärkere Beachtung von Narrativen spricht (siehe Melchior und Schürz 2015).

Abb. 2 zeigt die Selbsteinschätzung von Befragten in Bezug auf ihre Vermögensposition. Eine große Mehrheit der Menschen verortet sich fälschlich in der Mitte der Verteilung. Kaum jemand in Österreich glaubt, dass der eigene Haushalt in die Gruppe der vermögendsten $20 \%$ fällt. Und kein Einziger der reichsten $10 \%$ schätzt sich dem obersten Zehntel zugehörig. Die Positionen der obersten $10 \%$ sind nach Einschätzung der Vermögendsten vakant. Zwar ordnen sich auch dem untersten Dezil der Vermögensverteilung, d.h. den $10 \%$ mit den niedrigsten Vermögen, weniger Haushalte zu, als dort tatsächlich zu finden sind, doch deren Zahl ist viel geringer.

Die Mitte ist folglich in den Selbstwahrnehmungen überproportional besetzt (siehe Fessler und Schürz 2016). Der Begriff „Mitte“, der im politischen Diskurs oft als Zielreferenz bemüht wird, erlaubt fast allen Menschen sich angesprochen zu fühlen.

Durch das Entstehen einer dritten sozialen Schicht nach dem 2. Weltkrieg, die Immobilienvermögen hat, erhöhte sich nicht nur die Mobilität zwischen den Schichten, sondern damit einhergehend stiegen auch die Begründungsnotwendigkeiten und die Zahl der möglichen Narrative zu Reichtum. Es gab nun ab Mitte des 20. Jahrhundert eine Mittelschicht zwischen Arm und Reich mit einem starken Bezug zu Privatei- 


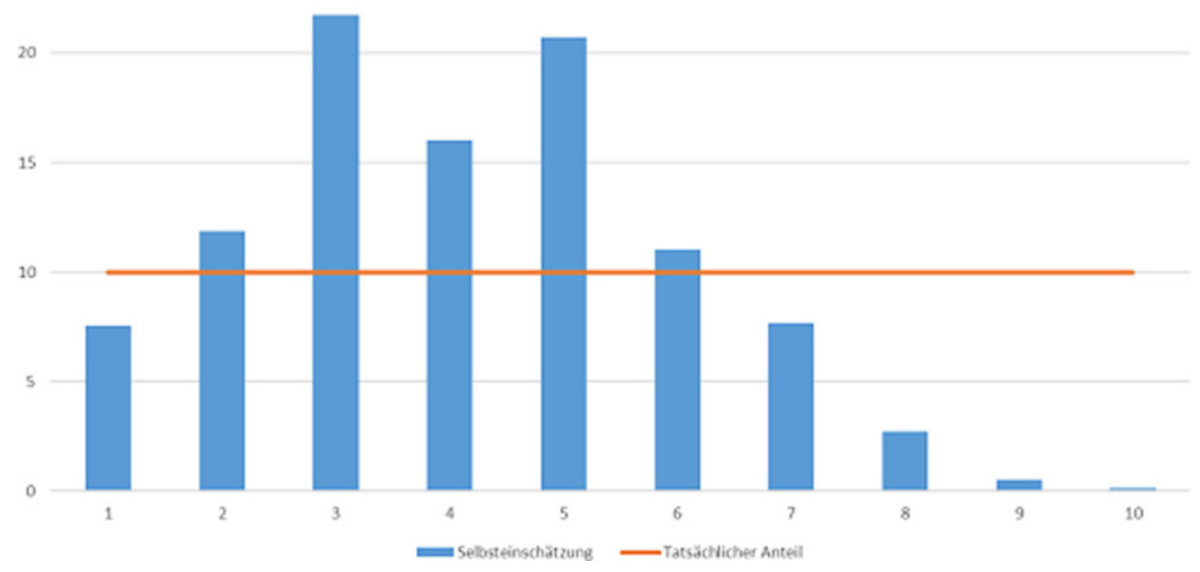

Abb. 2 Selbsteinschätzung zur Position in der Vermögensverteilung. Quelle: HFCS Austria 2017, OeNB

gentum. In Folge musste gerechtfertigt werden, wer sozial auf- und wer absteigen soll. Die Dreiteilung der Gesellschaft benötigte neue Begründungsnotwendigkeiten und veränderte Narrative zu Überreichtum (Schürz 2019).

Der Mitte werden Tugenden zugeschrieben und sie ist ein Sehnsuchtsort. Es ist ein konstruiertes Feld in der Gesellschaft, wo bestimmte Werte proklamiert werden. $\mathrm{Zu}$ diesen Werten zählt etwa Sicherheitsstreben. Immobilieneigentümer haben einen Ort, an welchem niemand ohne Zustimmung eindringen darf und an dem ihre eigenen Regeln gelten. Immobilien bilden daher eine besondere Vermögensform.

Gerade der Wert des eigenen Hauses wird nicht nur nach Geld, sondern nach ideellen Werten bemessen (siehe Fessler und Schürz 2021a, b). Immobilien sind zumeist mit Gefühlen verbunden. Häuser haben eine Geschichte und diese ist oft mit dem Lebensweg ihrer Eigentümer verknüpft. Es kann sich um das Elternhaus handeln, wo die Kindheit verbracht wurde oder um ein mühsam selbsterbautes Haus. Jedenfalls ist die Historie des Eigenheims vielfach gekennzeichnet von Lebensträumen. Während Finanzvermögen in Steueroasen verschoben werden kann, betont selbstgenutztes Immobilieneigentum ideelle Werte wie Sesshaftigkeit, Sicherheit und Kontinuität im Leben.

Damit hängt zusammen, dass sich auch sehr Vermögende als Teil der Mitte sehen. So machen sie implizit die vorgeblichen Tugenden der Mitte für sich selbst geltend. Und sie machen sich durch die Bezugnahme auf Immobilieneigentum kleiner. Ihr Reichtum wird ohnedies gerne über Luxusvillen veranschaulicht. Vergessen dabei wird, dass die Immobilien nur einen kleinen Bruchteil ihres Vermögens ausmachen.

Und Menschen verorten sich nicht zuletzt in der gesellschaftlichen Mitte, weil Mäßigung ein hegemoniales Narrativ, nicht zuletzt beim Konsum ist. In keiner Debatte wird für Maßlosigkeit plädiert, mag sie auch gelebt werden. Gepredigt wird vielmehr gegen Habgier, obzwar eine unbegrenzte Vermögensakkumulation vielleicht das Erwerbsstreben motiviert. Reichtum hat im Gegensatz zu Armut keine 
Abb. 3 Spenden in \% des Einkommens. Quelle: HFCS Austria 2017, OeNB

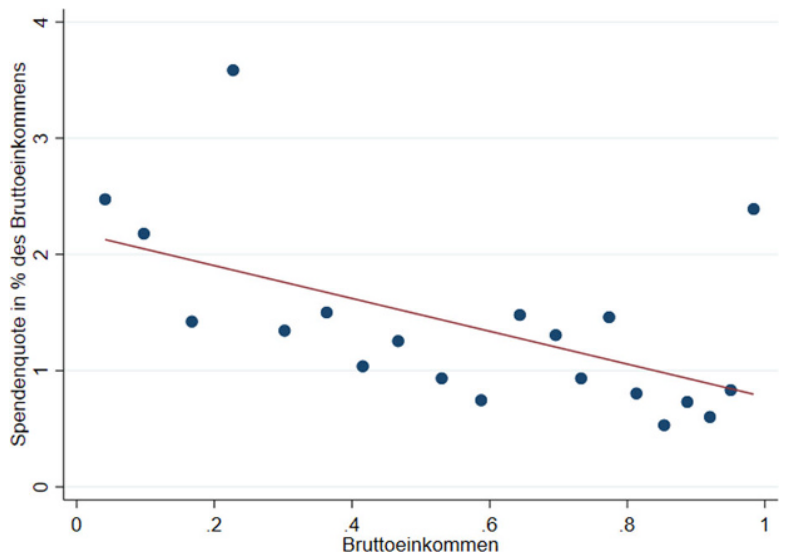

absolute Grenze. Und dies weist auf eine Vielzahl von Kombinationen an Werten und materieller Privilegierung hin. Smith hatte die Reichen, wie zuvor besprochen, keineswegs als besonders tugendhafte Menschen betrachtet. Er hatte die Vermögenden in seinem Werk charakterlich nicht ausgezeichnet. Reiche Menschen können im business as usual maßlose Gier leben und gleichzeitig sich wertorientiert in der Mitte positionieren und bescheiden konsumieren. Die Mitte als Aufstiegs- und Abstiegsort in der Gesellschaft ist Nährboden sozialer Nähe und beeinflusst Narrative zu Mäßigung, Verdienst und Tugend.

Die Daten des HFCS-Austria erlauben auch eine empirische Betrachtung der Spendentätigkeit in Relation zu Einkommen und Vermögen. Das vermutlich überraschende Ergebnis ist folgendes: Menschen mit niedrigem Einkommen spenden in Relation zur Höhe ihres Einkommens und ihres Vermögens mehr als reiche Menschen (siehe Abb. 3):

Während die Philanthropie als herrschaftssicherndes Instrument in Narrativen gut einsetzbar ist, bleibt es um das wohltätige Tun armer Menschen still. Es gibt keinen Sermon zur Philanthropie armer Menschen und allein die Idee einer Spendengala für ihre wohltätigen Beiträge wäre irritierend. Der Fokus auf hohen absoluten Geldbeträgen, etwa einer Millionenspende, oder die Giving Pledge Initiative von Milliardären verdrängt die kleinen Spendenbeiträge ärmerer Menschen in der wissenschaftlichen und medialen Beachtung.

\section{Eitelkeit der Reichen}

Michel de Montaigne hatte im 16. Jahrhundert in seinen Essais in Über die Eitelkeit geschrieben: „Jedes eifrige Bedachtsein auf Reichtum riecht nach Geiz - selbst in Form freigebigen Spendens, wenn es allzu geplant und berechnend ist. Der Reichtum verdient es nicht, daß man sich seinetwegen abmüht.“ (Montaigne 1999, S. 480).

Der reiche Mann in der Darstellung von Adam Smith ist ein eitler Geck. Eitel zu sein, wird nie in einem guten Wortsinn verwendet, schreibt Smith. Eitelkeit aber ,ist von vielen liebenswerten Tugenden begleitet, von Menschlichkeit, Höflichkeit, von 
einem Verlangen überall im Kleinen gefällig zu sein, ja manchmal von wirklichem Edelmut in großen Dingen, einem Edelmut jedoch, den sie oft in glänzenden Farben darzustellen liebt, als sie nur irgend vermag.“ (Smith 2010, S. 421). Die „Falschheiten des Eitlen sind alles unschuldige Falschheiten, ihr Zweck ist es den Betreffenden selbst zu erhöhen, nicht andere zu erniedrigen. “ (Smith 2010, S. 420).

Die eigene Großzügigkeit wird von eitlen Menschen in glänzenden Farben dargestellt, denn die Reichen wollen allseits geachtet werden. Ihre Lebensführung ist auf Vermögensanhäufung ausgerichtet, doch diese dient ihnen zur Darstellung. Vermögensmehrung ist kein Ziel an sich. Menschen streben nach Reichtum, um die Bewunderung anderer Menschen zu erlangen. Dies sei der Grund, warum Reiche ihren Reichtum lieben. Denn ansonsten würde gelten: „Wenn ich Reichtum zu erlangen suchte, schiene mir dieser Weg zu lang." (Montaigne 1999, S. 477). Und Montaigne hatte resignativ unterschieden: „Jeder von uns trägt das Seine zur Verderbnis der Zeit bei: nach Maßgabe ihrer jeweiligen Macht, die einen Treuseligkeit und Verrat, die anderen Ungerechtigkeit, Gottlosigkeit und Habsucht, Tyrannei und Grausamkeit - und die Schwächsten wiederum (zu denen ich gehör) Torheit, Eitelkeit und Nichtstun." (Montaigne 1999, S. 476).

Diese Form von Eitelkeit unterscheidet sich aber von jener Smiths beträchtlich:

Der reiche Mann rühmt sich seines Reichtums, weil er fühlt, daß dieser naturgemäß die Aufmerksamkeit der Welt auf ihn lenkt und daß die Menschen geneigt sind an all jenen Gemütsbewegungen gerne teilzunehmen, welche die Vorteile seiner Situation ihn so leicht einflößen müssen. (Smith 2010, S. 78)

Bei Adam Smith erfolgt eine irrationale Zuspitzung des Gefühls der Bewunderung. Es ist nicht einmal mehr der Reichtum allein, der Bewunderung findet, sondern auch die damit verbundene Anmaßung und Eitelkeit:

Es gibt kaum einen Menschen, der nicht den Reichen und Vornehmen bei gleichem Grade des persönlichen Verdienstes mehr achten würde als den Armen und Niedrigen. Von den meisten Menschen wird sogar die Anmaßung und Eitelkeit der ersteren weit mehr bewundert als das wirkliche und echte Verdienst der letzteren. (Smith 2010, S. 95).

Es seien „,nicht die Bequemlichkeit oder das Vergnügen“, welche Menschen in ihrem Streben nach Reichtum leiten. Der zentrale Vorzug von Reichtum sei: ,daß man mit Sympathie, Wohlgefallen und Billigung" auf sie achtet (Smith 2010, S. 78). Der Reiche will wohlwollend betrachtet werden und das Interesse von uns soll seiner Person und nicht nur seinem Vermögen gelten. Bei Adam Smith freut es die Reichen, dass sie uns in Fantasien beschäftigen. Indirekt nimmt demnach ein eitler Reicher an unserem Leben Anteil. Er will, dass wir seine Vergnügungen befürworten. Dafür benötigen wir aber unsere Einbildungskraft. In der sozialen Wirklichkeit treffen wir Reiche kaum und haben keine konkreten Vorstellungen zu ihrem Leben. Erst die Einbildungskraft erlaubt uns, auch das Missgeschick der über einem stehenden Menschen voll Bedauern zu betrachten.

Wohlwollen bezeichnet bei Adam Smith den Bereich des Altruismus. Dieser Bereich ist ein kleiner, denn viel an Wohlwollen bringen die Menschen füreinander wahrlich nicht auf. Adam Smith präsentierte in seiner Theorie der ethischen Gefüh- 
le das viel zitierte Gedankenexperiment eines gewaltigen Erdbebens in China. Die Reaktion eines human gesinnten Menschen in Europa wäre im ersten Moment Trauer, dann würde er trübselige Überlegungen zur Eitelkeit alles menschlichen Tuns anstellen, und später vielleicht zu den negativen Folgen für den Handel räsonieren. Und nach all diesen Gedanken würde er in Ruhe zu seinen Geschäften und Vergnügungen zurückkehren. Die kleinste Kleinigkeit, die ihn selbst betrifft, würde ihn mehr beunruhigen als der vollständige Untergang des chinesischen Reiches:

Das Bewußtsein, daß er morgen seinen kleinen Finger verlieren müßte, würde ihn schon heute Nacht nicht schlafen lassen; dagegen wird er bei dem Untergang von hundert Millionen seiner Brüder mit der tiefsten Seelenruhe schnarchen. (Smith 2010, S. 214).

Menschen lässt das Elend anderer Menschen oft recht gleichgültig. Hingegen wird sichtbare Armut als unverschämt empfunden: „Die Glücklichen und Stolzen staunen über die Unverschämtheit menschlichen Elends und wundern sich, daß dieses es wagen könne, sich vor ihnen zur Schau zu stellen, und daß es sich herausnehme, mit dem ekelhaften Anblick seiner Not die Heiterkeit ihres Glücks zu stören. " (Smith 2010, S. 79).

Wenn Menschen Leid hingegen nicht sehen, fehlt ihnen die Anteilnahme, denn „unbeachtet kommt und geht der arme Mann“ (Smith 2010, S. 79). Diese Unsichtbarkeit entspräche auch der gesellschaftlichen Stellung der Superreichen. Auch diese kleine Gruppe von Menschen wird kaum gesehen, doch sie wird viel beachtet. In diversen Narrativen erfolgt eine Annäherung an sie und über mediale Vermittlung gewinnen sie an Gestalt für die Bevölkerung. Und obzwar die Reichen gleichsam in einer anderen Welt leben, werden sie so zu Adressaten von wohlwollenden Gefühlen von uns. Ihr Lebensstil erscheint ,als beinahe die ideale Vorstellung eines vollkommenen und glücklichen Zustandes“" und: „Wie schade denken wir, wenn irgendetwas eine so angenehme Situation zerstören und verderben sollte. Ja, wir möchten gerade wünschen, daß jene Menschen unsterblich wären und es scheint uns hart, daß der Tod schließlich einem solch vollkommenen Genießen ein Ende setzen solle!" (Smith 2010, S. 80).

Das Streben nach Reichtum mag lächerlich angesichts des Todes wirken. Doch den Narrativen zur Endlichkeit menschlicher Existenz, dass letzte Hemd hat keine Taschen, eignet etwas bemüht Tröstliches. In unserer Einbildung erlaubt Vermögen wenigstens ansatzweise ein Stillen der Sehnsucht nach Transzendenz der Endlichkeit. Diese den Tod überschreitende Wirkung von Vermögen mag es sein, welche reiche Menschen in ihrem Tun motiviert.

\section{Conclusio}

Behauptungen zu einer rational begründeten herausragenden gesellschaftlichen Stellung der Reichen können deliberativ kritisiert werden. Die Rolle von Gefühlen folgt jedoch nicht den Argumentationsmustern einer idealen Sprechsituation. Eine Vielzahl von Narrativen wetteifert miteinander um Wirkungsmächtigkeit. Narrative zur Leistung von reichen Menschen, zu verdientem Reichtum und zu großzügigen Wohl- 
taten zählen zu jenen hegemonialen Erzählungen, welche die Akzeptanz von Vermögenskonzentration stärken sollen. Sie wären rational leicht zu beeinspruchen. In Erbschaften stecken keine Leistungen der Erben. Privateigentum hätte inhärente Grenzen, die unbedingt debattiert werden müssten. Und Großzügigkeit wäre in Relation zu den vorhandenen Mitteln zu betrachten.

Doch Narrative werden von Menschen nicht rational überprüft und es erfolgt kein empirischer Datencheck. Narrative greifen in Gefühlsmuster ein. Ob Reichtum verdient oder unverdient ist, spielt für Menschen eine nachgeordnete Rolle; ob die Reichen tugendhaft oder lasterhaft sind, ist nicht entscheidend für ihre Wahrnehmung. Wichtig sind Narrative, die an ein reichenfreundliches Gefühl der Bewunderung anknüpfen können. Auch in einer sozial partiell durchlässigen Gesellschaft behält die Bewunderung der Reichen ihre Funktionalität für die sich vertiefende Vermögenskonzentration.

Funding Open access funding provided by Vienna University of Economics and Business (WU).

Open Access Dieser Artikel wird unter der Creative Commons Namensnennung 4.0 International Lizenz veröffentlicht, welche die Nutzung, Vervielfältigung, Bearbeitung, Verbreitung und Wiedergabe in jeglichem Medium und Format erlaubt, sofern Sie den/die ursprünglichen Autor(en) und die Quelle ordnungsgemäß nennen, einen Link zur Creative Commons Lizenz beifügen und angeben, ob Änderungen vorgenommen wurden.

Die in diesem Artikel enthaltenen Bilder und sonstiges Drittmaterial unterliegen ebenfalls der genannten Creative Commons Lizenz, sofern sich aus der Abbildungslegende nichts anderes ergibt. Sofern das betreffende Material nicht unter der genannten Creative Commons Lizenz steht und die betreffende Handlung nicht nach gesetzlichen Vorschriften erlaubt ist, ist für die oben aufgeführten Weiterverwendungen des Materials die Einwilligung des jeweiligen Rechteinhabers einzuholen.

Weitere Details zur Lizenz entnehmen Sie bitte der Lizenzinformation auf http://creativecommons.org/ licenses/by/4.0/deed.de.

\section{Literatur}

Adorno, Theodor. 2003. Minima Moralia. Reflexionen aus dem beschädigten Leben. Gesammelte Schriften, Bd. 4. Suhrkamp.

Aristoteles. 1999. Rhetorik. Reclam.

Brown, Peter. 2017. Der Schatz im Himmel. Der Aufstieg des Christentums und der Untergang des Römischen Weltreichs. Klett-Cotta.

Bundesministerium für Arbeit und Soziales. 2013. 4. Armuts- und Reichtumsbericht. https://www.armutsund-reichtumsbericht.de/DE/Bericht/Archiv/Der-vierte-Bericht/vierter-bericht.html. Zugegriffen: 06.09.2021

Callahan, David. 2017. The Givers: wealth, power and philanthropy in a new gilded age.

Evensky, Jerry. 2005. Adam Smith's theory of moral sentiments. On morals and why they matter to a liberal society of free people and free markets. Journal of Economic Perspectives 19(3):109-130.

Fessler, Pirmin, und Martin Schürz. 2016. Zur Mitte in Österreich. In Sozialbericht 2016, 270-291. Bundesministerium für Soziale Angelegenheiten.

Fessler, Pirmin, und Martin Schürz. 2017. Zur Verteilung der Sparquoten in Österreich. Monetary Policy and Economy 3/2017:13-33.

Fessler, Pirmin, und Martin Schürz. 2018. Private wealth across European countries: the role of income, inheritance and the welfare state. Journal of Human Development and Capabilities 19(4):521-549. https://doi.org/10.1080/19452829.2018.1507422.

Fessler, Pirmin, und Martin Schürz. 2021a. Homeownership_a key narrative to explain wealth inequality? In Media and Inequality, Hrsg. Steve Schifferes, Sophie Knowles. Routledge. forthcoming. 
Fessler, Pirmin, und Martin Schürz. 2021b. Housing and the American Dream: Is a house still a home? : Institute for New Economic Thinking. ineteconomics.org.

Frevert, Ute. 2013. Vergängliche Gefühle. Wallstein.

Gabriel, Markus. 2020. Moralischer Fortschritt in dunklen Zeiten. Ullstein.

Hayek, August. 2005. Verfassung der Freiheit. Mohr Siebeck.

Mandeville, Bernard. 2014. Die Bienenfabel. Suhrkamp.

Melchior, Josef, und Martin Schürz. 2015. Gerechtigkeitsurteile und Vermögensverteilung. Wirtschaft und Gesellschaft 41(2):199-232.

Montaigne, Michel. 1999. Essais. Die Andere Bibliothek.

Nussbaum, Martha. 2014. Politische Emotionen. Warum Liebe für Gerechtigkeit wichtig ist. Suhrkamp.

Nussbaum, Martha. 2016. Königreich der Angst. Gedanken zur aktuellen politischen Krise. Btb.

Obermeier, Otto-Peter. 2019. Moralisch fühlen, gierig handeln. Zur Aktualität von Adam Smiths „,Theorie der moralischen Gefühle "Der Blaue Reiter.

Piff, Paul. 2014. Wealth and the inflated self: class, entitlement, and narcissism. Personality and Social Psychology Bulletin 40(1):34-43.

Piketty, Thomas. 2014. Capital in the 21. Century. Harvard University Press.

Piketty, Thomas. 2020. Kapital und Ideologie. C.H. Beck.

Schürz, Martin. 2019. Überreichtum. Frankfurt New York: Campus.

Schürz, Martin. 2021. Ungerechtfertigte Vermögensverhältnisse. In Wirtschaft und Gesellschaft 2/2021. Rezensionsartikel zu Thomas Piketty Kapital und Ideologie.

Sen, Amartya. 2010. Adam Smith and contemporary world. Erasmus Journal of Economics and Philosophy 3(1):50-67. https://doi.org/10.23941/ejpe.v3i1.39.

Shklar, Judith. 1992. Über Ungerechtigkeit. Erkundungen zu einem moralischen Gefühl. Rotpunkt.

Shklar, Judith. 2013. Der Liberalismus der Furcht. Berlin: Matthes \& Seitz.

Shklar, Judith. 2014. Ganz normale Laster. Berlin: Matthes \& Seitz.

Simmel, Georg. 1989. Philosophie des Geldes. Suhrkamp.

Smith, Adam. 2009. Wohlstand der Nationen. Anaconda.

Smith, Adam. 2010. Theorie der ethischen Gefühle. Meiner.

Smith Ochoa, Christopher. 2019. Trivilazing inequality by narrating facts: a discourse analysis of contending storylines in Germany. In Critical Policy Studies, 1-20.

Therborn, Göran. 2017. Dynamics of inequality. New Left Review 103:1-19.

Wiesing, Lambert. 2015. Luxus. Suhrkamp.

Yildiz, Taylan, Frank Gadinger, und Smith Christopher. 2018. Narrative Legitimierung: Exekutive, repräsentative und subversive Erzählstrategien in der Überwachungskontroverse. Leviathan 46(1):135-162. 